\title{
Multiplex RT-PCR assay to differentiate genotypes of porcine reproductive and respiratory syndrome virus in swine
}

\author{
Nam T. Nguyen ${ }^{1}$, Giao N. P. Trinh ${ }^{2}$, The N. Nguyen ${ }^{1}$, \& Phat X. Dinh ${ }^{1 *}$ \\ ${ }^{1}$ Department of Biotechnology, Nong Lam University, Ho Chi Minh City, Vietnam \\ ${ }^{2}$ Faculty of Animal Science and Veterinary Medicine, Nong Lam University, Ho Chi Minh City, Vietnam
}

\author{
ARTICLE INFO \\ Research Paper \\ Received: August 19, 2019 \\ Revised: September 19, 2019 \\ Accepted: November 22, 2019 \\ Keywords \\ Differential diagnosis \\ Multiplex reverse transcriptase PCR \\ (multiplex RT-PCR) assay \\ Porcine reproductive and respiratory \\ syndrome virus (PRRSV) \\ Specific primers

\section{${ }^{*}$ Corresponding author} \\ Dinh Xuan Phat \\ Email: dinhxuanphat@hcmuaf.edu.vn
}

\begin{abstract}
Porcine reproductive and respiratory syndrome (PRRS) is one of the most economically important diseases to swine industry worldwide. Due to the heterogeneity of field isolates, accurate detection of the PRRS virus is a diagnostic challenge. Recently, coinfection with NA-PRRSV, EU-PRRSV and HP-PRRSV isolates continuously increases in many countries, resulting in a significant impact on PRRSV diagnostics and disease control on farms. To facilitate rapid diagnosis and reliable discrimination of NA-PRRSV, EU-PRRSV and HP-PRRSV, a multiplex RT-PCR assay was established with three pairs of primers targeting highly conservative regions of nsp2 gene with predicted multiplex RT-PCR products of $364 \mathrm{bp}, 161 \mathrm{bp}$ and $259 \mathrm{bp}$, respectively. The primer pairs were optimized to be highly specific for PRRSV genotypes and were able to detect the target gene at the limit of $10^{2}$ copies/uL for each gene. Clinical samples were used to evaluate this multiplex RT-PCR in parallel with a commercial realtime RT-PCR kit. Results showed over $95.2 \%$ (20/21 samples) agreement between the mRT-PCR and the real-time RT-PCR kit. Hence, it indicated that this multiplex RT-PCR could be useful for rapid and differential diagnosis of NA-PRRSV, EU-PRRSV and HP-PRRSV in swine farms.
\end{abstract}

Cited as: Nguyen, N. T., Trinh, G. N. P., Nguyen, T. N., \& Dinh, P. X. (2019). Multiplex RT-PCR assay to differentiate genotypes of porcine reproductive and respiratory syndrome virus in swine. The Journal of Agriculture and Development 18(6), 8-13.

\section{Introduction}

Porcine Reproductive and Respiratory Syndrome (PRRS) or "blue ear" disease is a highly contagious viral disease that was first recognized in North America in the late 1980s, followed in Europe and Asia. The main clinical signs of this disease are late-term reproductive failures in sows as well as reduction in rate of growth and increased mortality rate in all age groups due to severe pneumonia (Terpstra et al., 1991; Rossow et al., 1994). Thus, PRRS causes significantly economic loss in most swine-producing countries worldwide.

The etiological agent of PRRS is porcine reproductive and respiratory syndrome virus (PRRSV) which belongs to the Nidovirales order, Arterivirus genus and Arteriviridae family (Cavanagh, 1997). Porcine Reproductive and Respiratory Syndrome virus is an enveloped, single positive-stranded RNA virus with a genome ranging from 13 to $15 \mathrm{~Kb}$ in length, which contains at least 10 open reading frames (ORFs). The ORF1a and ORF1b comprise more than $75 \%$ of the genome and encode non-structural polyproteins responsible for viral replication (Kappes \& Faaberg, 2015). The ORF2, ORF3 and ORF4 encode for the minor structural surface proteins GP2, GP3 and GP4, respectively and together these proteins form a trimeric complex that is heavily $\mathrm{N}$-glycosylated and functions in viral entry (Das et al., 2010, 2011). Furthermore, ORF5, 
ORF6 and ORF7 encode for the major structural surface proteins GP5, matrix (M) and nucleocapsid $(\mathrm{N})$, respectively. Besides, the N-glycosylated GP5, a unglycosylated protein is translated from an alternative reading frame, designated ORF5a (Johnson et al., 2011; Kappes \& Faaberg, 2015).

Phylogenetic analysis of this causative agent isolates worldwide indicates clearly existence of two major genotypes, the European genotype (EU-PRRSV or type 1) and North American genotype (NA-PRRSV or type 2) with Lelystad and VR-2332 as prototypical strains, respectively (Meng et al., 1995). Both types share an approximately $60 \%$ sequence identity at the nucleotide level (Nelsen et al., 1999). Until May 2006, a new variant of PRRSV emerged, later defined as a highly pathogenic form of PRRSV (HPPRRSV), and severely impacted the pig industry with the death of more than two million pigs in South China (Tian et al., 2007). At the molecular level, common characteristics of all highly virulent PRRSV isolates were usually demonstrated by a unique discontinuous deletion of 30 amino acids (aa) in the non-structural protein 2 (nsp2) that has been proposed as the genetic marker of the HP-PRRSV (Tian et al., 2007).

The diagnosis of PRRSV has been accomplished typically by virus isolation, immunohistochemistry, serological methods, or conventional reverse transcriptase PCR (RT-PCR) (Mengeling et al., 2000). The drawbacks of these methods are time-consuming, labor-intensive, potential for cross-contamination and high costs. Obviously, RT-PCR assay is highly specific and sensitive, however it is difficult for PRRSV genotype or strains discrimination. Meanwhile, the co-infection among different PRRSV genotypes has been increasingly reported, resulting in significant challenges on PRRSV diagnostics and management. Therefore, a rapid and reliable multiplex RT-PCR assay for differential diagnosis of NA-PRRSV, EU-PRRSV and HP-PRRSV strains in this study is quite useful for routine diagnosis of PRRS in swine farms.

\section{Materials and Methods}

\subsection{Nucleic acid for reaction controls and clin- ical samples}

The RNA genome of NA-PRRSV (VR-2332, Genbank ID U87392), EU-PRRSV (DV, similar with Lelystad strain, Genbank ID M96262) and HP-PRRSV (JAX1, Genbank ID EF112445) strains used in this research were extracted from live-attenuated vaccines, includes Ingelvac PRRS MLV vaccine (Boehringer, Germany), Porcilis PRRS vaccine (Intervet, Netherland) and a vaccine product of Hoa Nong Company (China), accordingly. Viral RNA was extracted by TRIzol Plus RNA Purification kit (Cat\#12183555, Invitrogen, USA).

Genetic material of other viruses and bacteria, including Classical swine fever virus (CSFV), Pseudorabies virus (PRV), Haemophilus parasuis, Mycoplasma hyopneumoniae, Streptococcus suis, Staphylococcus aureus and DNA of pig used for evaluation of the specificity of the mRT-PCR were provided by the Sanphar Vietnam laboratory (Erber group, Austria) and IDT (United States).

Regarding clinical samples, a total of 21 serum $(n=10)$ and lung tissue samples $(n=11)$ were collected from swine farms in Dong Nai and Binh Duong provinces and were used as field sample to evaluate the potential application of the established mRT-PCR.

\subsection{Primer design}

Three pairs of primers distinguishing highly pathogenic PRRSV (HP) versus PRRSV type 1 (EU) and PRRSV type 2 (NA) were designed in nsp2 gene segment by MPRIMER software. The nucleotide sequence of target gene obtained from NCBI (Table 1), validated by NCBI BLAST, OligoAnalyzer 1.0.2 software. The annealing temperature and the size of the amplified product were adjusted for clear segregation in electrophoresis. Primers were synthesized by IDT (Integrated DNA Technologies, USA).

\subsection{RT-PCR optimization}

Before setting up mRT-PCR, all primers were evaluated for functioning in single RT-PCRs according to the predicted annealing temperature and regular RT-PCR protocol. The sRT-PCR was performed in a $30 \mathrm{uL}$ reaction containing $15 \mu \mathrm{L}$ PCRBIO 2X One Step RT-PCR kit (Cat\#PB105210, PCR Biosystems, England), 1.5 $\mu \mathrm{L}$ RTase $20 \mathrm{X}, 0.5 \mu \mathrm{M}$ of each primer, $2 \mu \mathrm{L}$ of RNA template, and nuclease-free water to adjust the final volume to $20 \mu \mathrm{L}$. Nuclease-free water was also used as a negative control for all PCRs. The RT-PCR was carried out under the 
Table 1. Primers used in this study

\begin{tabular}{llc}
\hline Name & Sequences (5'-3') & size(bp) \\
\hline \multirow{2}{*}{ NA364 } & F: CCTGGGTGTGGTGTTTCT & \multirow{2}{*}{364} \\
& R: ATGGCGACGTGTTAGGCT & \\
\multirow{2}{*}{ HP259 } & F: ATCATGACCAGGTGTGCTTG & \multirow{2}{*}{259} \\
& R: TGTTCGGTTGTCTGATTCGC & \\
\multirow{2}{*}{ EU161 } & F: GGTGGAATGTACTCCATCCG & \multirow{2}{*}{161} \\
& R: CTTGGAGTTCACGAAGGTGT & \\
\hline
\end{tabular}

following conditions: a reverse transcription step at $45^{\circ} \mathrm{C}$ for $10 \mathrm{~min}$, reverse transcriptase inactivation and pre-denaturation at $95^{\circ} \mathrm{C}$ for $2 \mathrm{~min}$, followed by 40 cycles consisting of denaturation at $95^{\circ} \mathrm{C}$ for $15 \mathrm{sec}$, annealing at optimal temperature for $30 \mathrm{sec}$, and extension at $72^{\circ} \mathrm{C}$ for $30 \mathrm{sec}$, Ten $\mu \mathrm{L}$ of amplified products were separated on $2 \%$ agarose gel electrophoresis in 0.5X Tris-BorateEDTA (TBE) with Midori Green Advance DNA stain (Cat\#AG10, Nippon) and 1 kb Plus DNA ladder (Cat\#10787018, Invitrogen) as the molecular weight markers to indicate the sizes of the amplified products. The effects of annealing temperature ranging from $52^{\circ} \mathrm{C}$ to $60^{\circ} \mathrm{C}$ were also determined experimentally.

\subsection{Optimization of multiplex RT-PCR assay}

To establish a functional mRT-PCR, several aspects need to be examined such as annealing temperatures, primer concentration, primer ratio,... After several rounds of optimization, the final primer mix with a ratio of $0.5 \mu \mathrm{M}(\mathrm{NA}): 0.5$ $\mu \mathrm{M}$ (EU): $0.5 \mu \mathrm{M}$ (HP) was accordingly achieved. The final multiplex RT-PCR mixtures contained $15 \mu \mathrm{L}$ PCRBIO 2X One Step RT-PCR kit, $1.5 \mu \mathrm{L}$ Rtase $20 \mathrm{X}, 0.5 \mu \mathrm{M}$ of each of the three primers, $2 \mu \mathrm{L}$ of RNA template and nuclease free water up to $20 \mu \mathrm{M}$ in total. Thermal cycling conditions and gel electrophoresis were similar to the one described above for sRT-PCR.

\subsection{Specificity and detection limit}

To evaluate the specificity of the mRT-PCR assay, DNA of different viruses and bacteria that are commonly found in swine farms and potentially contaminate during sampling process were used as unrelated template in reactions. For confirmation of the mRT-PCR products, DNA bands were recovered from low melting agarose gel (Cat\#BP165-25, Thermo Fisher Scientific, USA), using phenol-chloroform method (Green
\& Sambrook, 2012), and sequenced by Nam Khoa Biotek company. Later on, the resultant nucleotide sequences of the mRT-PCR products were aligned with reference sequences in Genbank using BLAST tool to verify the specificity of the mRT-PCR.

Concerning the detection limit, the purified RNA templates were first quantified by Realtime PCR (PowerCheckTM PRRSV Realtime PCR, Kogenebiotech, Korea) then were diluted 10-fold serially in nuclease-free water. The diluted template were further used to determine the detection limit of the established mRT-PCR.

\subsection{Application in diagnosis of clinical sam- ples}

The potential application of the established mRT-PCR assay in routine diagnosis of PRRSV for field samples was determined by head to head comparison with the results produced by commercial Realtime PCR assay. A total of 21 clinical samples were analyzed in this study.

\section{Results and Discussion}

\section{1. mRT-PCR establishment}

In sRT-PCR reactions to examine the general conditions for the assay, gel electrophoresis analysis showed the correct product sizes as predicted, including $364 \mathrm{bp}$ for NA PRRSV, $259 \mathrm{bp}$ for HP PRRSV, and $161 \mathrm{bp}$ for EU PRRSV. The results also indicated that 3 pairs of primers worked properly in the annealing temperature range of $52^{\circ} \mathrm{C}-60^{\circ} \mathrm{C}$ (data not shown), and the $54^{\circ} \mathrm{C}$ was chosen for mRT-PCR in this study. The primer concentration and ratio were $1 \mathrm{NA}: 1 \mathrm{HP}: 1 \mathrm{EU}$ at $0.5 \mu \mathrm{M}$ per reaction. Additionally, the three products were clearly visible and distinguishable from each other, no visible band was found in the lane of negative control (Figure 1). 


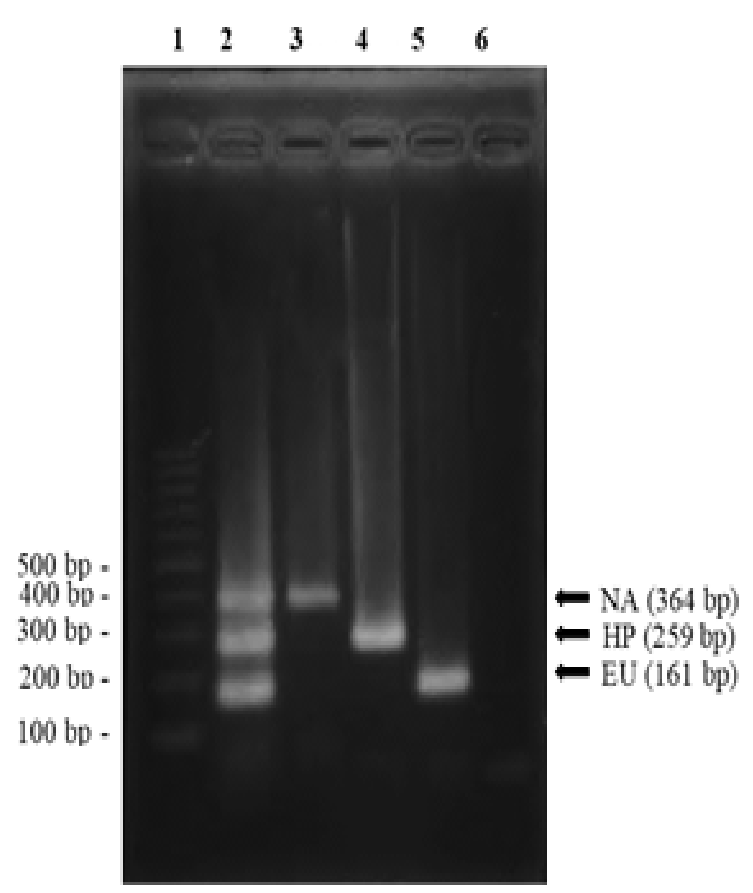

Figure 1. Products of sPCRs and mPCR. (1): DNA ladder $1 \mathrm{~Kb}$ plus, (2): mRT-PCR of all three targets, (3): NA-PRRSV 364 bp, (4): HP-PRRSV 259 bp, (5): EU-PRRSV 161 bp, (6): negative control with pure water. The thermal cycling conditions were $94^{\circ} \mathrm{C} / 5 \mathrm{~min} ; 35$ cycles of $94^{\circ} \mathrm{C} / 30 \mathrm{sec}, 54^{\circ} \mathrm{C} / 30 \mathrm{sec}$ and $72^{\circ} \mathrm{C} / 40 \mathrm{sec}$, a final extension at $72^{\circ} \mathrm{C} / 5 \mathrm{~min}$; Gel electrophoresis was $2 \%$ agarose and performed at $90 \mathrm{vol} / 25 \mathrm{~min}$.

\subsection{Specificity and detection limit of the mRT-PCR}

After the primer ratio and the thermal cycling conditions have been established. The specificity and the detection limit of the mRT-PCR were determined. Based on the routine usage of blood or serum in practical disease monitoring which can also contain different microbes due to septicemia or contamination, unrelated nucleic acid templates isolated from bacteria and/or viruses commonly found in pig farms were used in this experiment, including Staphylococcus aureus, Streptococcus suis, Mycoplasma hyopneumoniae, Pseudorabies virus, Classical swine fever virus and genomic DNA from pigs. Results showed that the three primer pairs did not cross-react with any of these templates (Figure 2), indicating the established mRT-PCR has expected specificity. We then evaluated the limit of detection of the mRTPCR by performing the assay with a set of se-

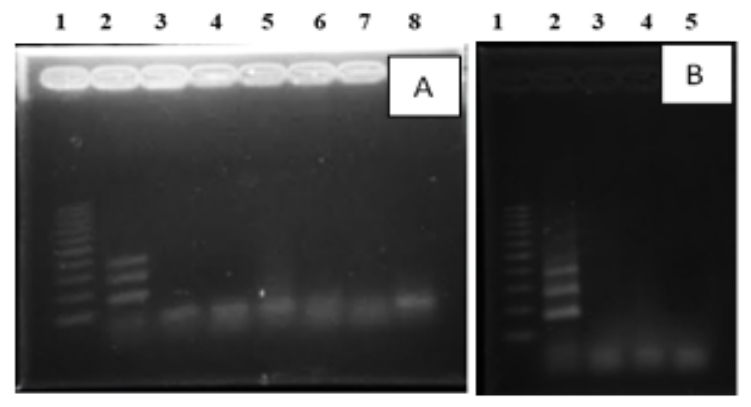

Figure 2. Specificity of mRT-PCR. A. (1): ladder $1 \mathrm{~Kb}$ plus, (2): positive control, (3): negative control with pure water, (4): Streptococcus suis, (5): Staphylococcus aureus, (6): Mycoplasma hyopneumoniae, (7): Pseudorabies virus, (8): Classical swine fever virus. B. (1): ladder $1 \mathrm{~Kb}$ plus, (2): positive control, (3): pig DNA, (4): Haemophilus parasuis, (5): negative control with pure water. The thermal cycling conditions were: $94^{\circ} \mathrm{C} / 5 \mathrm{~min} ; 35$ cycles of $94^{\circ} \mathrm{C} / 30 \mathrm{~s}, 54^{\circ} \mathrm{C} / 30 \mathrm{~s}$ and $72^{\circ} \mathrm{C} / 40 \mathrm{~s}$, a final extension at $72^{\circ} \mathrm{C} / 5 \mathrm{~min}$; Gel electrophoresis was at $90 \mathrm{vol} / 25 \mathrm{~min}$.

rially 10 -fold diluted positive control templates with the copy numbers ranging from $2 \times 10^{3}$ to 2 $\mathrm{x} 10^{0}$ copies per reaction. As shown in Figure 3, three distinct DNA bands at expected size were observed at the concentration of $2 \times 10^{2}$ copies per reaction. It demonstrated that this mRTPCR could detect the viral genes at the limit of 200 copies/gene/reaction.

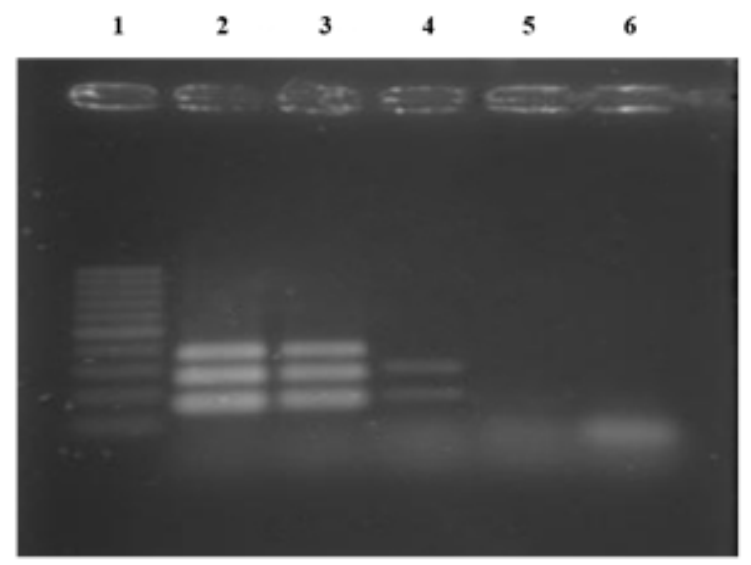

Figure 3. Detection limit of mRT-PCR. (1): ladder $1 \mathrm{~Kb}$ plus, (2-5): RNA template of NA PRRSV, HP PRRSV and EU PRRSV at $2 \times 10^{3}-2 \times 10^{0}$ copies/reaction, (6): negative control with pure water. The thermal cycling conditions were: $94^{\circ} \mathrm{C} / 5$ min; 35 cycles of $94^{\circ} \mathrm{C} / 30 \mathrm{sec}, 54^{\circ} \mathrm{C} / 30 \mathrm{sec}$ and $72^{\circ} \mathrm{C} / 40 \mathrm{sec}$, a final extension at $72^{\circ} \mathrm{C} / 5 \mathrm{~min}$; Gel electrophoresis was at $90 \mathrm{vol} / 25 \mathrm{~min}$. 
Table 2. Detection of clinical specimens by multiplex RT-PCR and Realtime RT-PCR

\begin{tabular}{|c|c|c|c|c|c|c|c|}
\hline \multirow{2}{*}{ Pig farm } & \multirow{2}{*}{ No. of specimens } & \multicolumn{3}{|c|}{ mRT-PCR } & \multicolumn{3}{|c|}{ Realtime RT-PCR } \\
\hline & & $\mathrm{NA}$ & $\mathrm{HP}$ & $\mathrm{EU}$ & $\mathrm{NA}$ & $\mathrm{HP}$ & $\mathrm{EU}$ \\
\hline \multirow{3}{*}{1} & 1.1 & + & + & - & + & + & - \\
\hline & 1.2 & - & + & - & - & + & - \\
\hline & 1.3 & - & + & + & - & + & + \\
\hline \multirow{3}{*}{2} & 2.1 & + & + & - & + & + & - \\
\hline & 2.2 & + & - & - & + & - & - \\
\hline & 2.3 & + & - & - & + & - & - \\
\hline \multirow{3}{*}{3} & 3.1 & - & + & - & - & + & - \\
\hline & 3.2 & + & + & - & + & + & - \\
\hline & 3.3 & - & - & + & - & - & + \\
\hline \multirow{3}{*}{4} & 4.1 & + & + & - & + & + & - \\
\hline & 4.2 & - & + & - & - & + & - \\
\hline & 4.3 & - & - & + & - & - & + \\
\hline \multirow{3}{*}{5} & 5.1 & + & - & - & + & - & - \\
\hline & 5.2 & - & + & + & - & + & + \\
\hline & 5.3 & + & - & - & + & - & - \\
\hline \multirow{3}{*}{6} & 6.1 & - & + & - & + & + & - \\
\hline & 6.2 & + & + & - & + & + & - \\
\hline & 6.3 & + & - & - & + & - & - \\
\hline \multirow{3}{*}{7} & 7.1 & - & + & + & - & + & + \\
\hline & 7.2 & - & - & + & - & - & + \\
\hline & 7.3 & - & + & - & - & + & - \\
\hline \multicolumn{2}{|c|}{ Concordance rate $\%$} & $\begin{array}{c}10 \\
90.9 \\
(10 / 11)\end{array}$ & $\begin{array}{c}13 \\
100 \\
(13 / 13)\end{array}$ & $\begin{array}{c}6 \\
100 \\
(6 / 6)\end{array}$ & 11 & 13 & 6 \\
\hline
\end{tabular}

This detection limit is comparable to the result reported by Xiao et al. (2014). In that study, the author used RT-PCR developed based on SYBR Green and TaqMan probe to discriminate highly pathogenic PRRSV against NA PRRSV, result of detection limit was $5 \times 10^{2}$ copies/ reaction. In addition, Balka et al. (2009) reported the detection limit in their Realtime RT-PCR assay to be approximately 10 copies/reaction. Other research provided that mRT-PCR for detection EU PRRSV, HP-PRRSV and NA PRRSV has an analytical sensitivity of less than 200 copies/reaction (Wernike et al., 2012). Therefore, detection limit of mRT-PCR achieved in this study was quite suitable for routine diagnosis.

\subsection{Detecting the presence of PRRS virus from clinical samples}

To examine the utility of the mRT-PCR for routine diagnosis of PRRSV genotypes circulating in swine farms which can help to guide the farm in choosing appropriate vaccines. A total of 21 samples including serum and tissues were used in parallel testing between the mRT-PCR and a commercial Realtime RT-PCR kit. Result summarized in Table 2 showed that the agreement between the mRT-PCR and realtime RT-PCR assays was $90.9 \%$ (10/11 samples), 100\% (13/13 samples) and $100 \%$ (6/6 samples), respectively for NA-PRRSV, EU-PRRSV and HP-PRRSV. Interestingly, co-infection among PRRSV strains were observed in these field samples. In details, there were 3 samples positive for both HP-PRRSV and EU-PRRSV, 6 samples positive for both HP-PRRSV and NA-PRRSV. It indicated that the mRT-PCR would be useful for differential diagnosis and/or determination of coinfection of various genotypes in a swine herd.

\section{Conclusions}

In summary, the established mRT-PCR was a convenient, rapid, sensitive and specific assay for the identification of different PRRSV genotypes. The size of amplified products is distinguishable. The method showed a feasible application in regular laboratory testing for viral surveillance 
and for effective control of the NA-PPRSV, EUPRRSV and HP-PRRSV strains in swine farms.

\section{References}

Balka, G., Hornyak, A., Balint, A., Benyeda, Z., \& Rusvai, M. (2009). Development of a one-step real-time quantitative PCR assay based on primer-probe energy transfer for the detection of porcine reproductive and respiratory syndrome virus. Journal of Virological Methods 158, 41-45.

Cavanagh, D. (1997). Nidovirales: A new order comprising Coronaviridae and Arteriviridae. Archives of $\mathrm{Vi}$ rology 142(3), 629-633.

Das, P. B., Dinh, P. X., Ansari, I. H., de Lima, M., Osorio, F. A., \& Pattnaik, A. K. (2010). The minor envelope glycoproteins GP2a and GP4 of porcine reproductive and respiratory syndrome virus interact with the receptor CD163. Journal of Virology 84(4), 1731-1740.

Das, P. B., Vu, H. L. X., Dinh, P. X., Cooney, J. L., Kwon, B., Osorio, F. A., \& Pattnaik, A. K. (2011). Glycosylation of minor envelope glycoproteins of porcine reproductive and respiratory syndrome virus in infectious virus recovery, receptor interaction, and immune response. Virology 410(2), 385-394.

Green, M. R., \& Sambrook J. (2012). Molecular cloning: A laboratory manual - Volume 1 ( $4^{\text {th }}$ ed.). New York, USA: Cold Spring Harbor Laboratory Press.

Johnson, C. R., Griggs, T. F., Gnanandarajah, J., \& Murtaugh, M. P. (2011). Novel structural protein in porcine reproductive and respiratory syndrome virus encoded by an alternative ORF5 present in all arteriviruses. Journal of General Virology 92(5), 1107-1116.

Kappes, M. A., \& Faaberg, K. S. (2015). PRRSV structure, replication and recombination: Origin of phenotype and genotype diversity. Virology 479-480, 475486.

Meng, X. J., Paul, P. S., Halbur, P. G., \& Lum, M. A. (1995). Phylogenetic analyses of the putative M (ORF 6 ) and N (ORF 7) genes of porcine reproductive and respiratory syndrome virus (PRRSV): Implication for the existence of two genotypes of PRRSV in the U.S.A. and Europe. Archives of Virology 140(4), 745-755.
Mengeling, W. L., \& Lager, K. M. (2000). A brief review of procedures and potential problems associated with the diagnosis of porcine reproductive and respiratory syndrome. Veterinary Research 31(1), 61-69.

Nelsen, C. J., Murtaugh, M. P., \& Faaberg, K. S. (1999). Porcine reproductive and respiratory syndrome virus comparison: Divergent evolution on two continents. Journal of Virology 73(1), 270-280.

Rossow, K. D., Bautista, E. M., Goyal, S. M., Molitor, T. W., Murtaugh, M. P., Morrison, R. B., Benfield, D. A., \& Collins, J. E. (1994). Experimental porcine reproductive and respiratory syndrome virus infection in one-, four-, and 10-week-old pigs. Journal of Veterinary Diagnostic Investigation 6(1), 3-12.

Terpstra, C., Wensvoort, G., \& Pol, J. M. A. (1991). Experimental reproduction of porcine epidemic abortion and respiratory syndrome (mystery swine disease) by infection with Lelystad vims: Koch's postulates fulfilled. Veterinary Quarterly 13(3), 131-136.

Tian, K., Yu, X., Zhao, T., Feng, Y., Cao, Z., Wang, C., Hu, Y., Chen, X., Hu, D., Tian, X., Liu, D., Zhang, S., Deng, X., Ding, Y., Yang, L., Zhang, Y., Xiao, H., Qiao, M., Wang, B., \& Gao, G. F. (2007). Emergence of fatal PRRSV variants: Unparalleled outbreaks of atypical PRRS in China and molecular dissection of the unique hallmark. PLoS One 2(6), e526.

Xiao, S., Chen, Y., Wang, L., Gao, J., Mo, D., He, Z., \& Liu, X. (2014). Simultaneous detection and differentiation of highly virulent and classical Chinese-type isolation of PRRSV by real-time RT-PCR. Journal of Immunology Research 2014, 1-7.

Wernike, K., Hoffmann, B., Dauber, M., Lange, E., Schirrmeier, H., \& Beer, M. (2012). Detection and typing of highly pathogenic porcine reproductive and respiratory syndrome virus by multiplex real- time RTPCR. PLos One 7(6), e38251. 